\title{
Freisetzung von Stoffwechselenzymen aus den Thrombocyten der Maus und des Meerschweinchens während der Blutgerinnung ${ }^{1)}$
}

\author{
Von R. Frieder ${ }^{2}$ ) und H. Mattenheimer \\ Departments of Biochemistry and Medicine, Rush-Presbyterian-St. Luke's Medical Center und Department of Biochemistry, \\ University of Illinois, College of Medicine, Chicago, Illinois, US A
}

(Eingegangen am 17. November 1970)

Der Einfluß der Blutgerinnung auf die Aktivitäten der Lactatdehydrogenase (EC 1.1.1.27), Malatdehydrogenase (EC 1.1.1.37), Aspartataminotransferase (EC 2.6.1.1), Alaninaminotransferase (EC 2.6.1.2) und $\gamma$-Glutamyltranspeptidase (EC 2.3.2.1) im Serum der Maus und des Meerschweinchens wurde untersucht. Bei beiden Spezies treten während der Gcrinnung Enzyme aus den Thrombocyten aus. Dieser Enzymaustritt hat ein solches Ausmaß, daß Aktivitätsbestimmungen von Enzymen im Serum in ihrer diagnostischen Aussagektaft eingeschränkt werden oder wertlos sind, falls die betreffenden Enzyme in hoher Aktivität in Thrombocyten vorhanden sind (wie Lactat- und Malatdehydrogenase). Es bestehen Anhaltspunkte dafür, daß. Enzyme niederen Molekulargewichts leichter austreten als solche mit höherem Molekulargewicht. Aus den Thrombocyten des Meerschweinchens wird bei der Gerinnung auch die streng strukturgebundene $\gamma$-Glutamyltranspeptidase freigesetzt. Sie geht bei der Gerinnung entweder in Lösung, oder es werden subzelluläre Partikel frei, an die das Enzym gebunden ist.

\section{Release of enzymes from thrombocytes during blood clotting of mouse and guinea pig}

The effect of blood clotting on the activities in serum of lactate dehydrogenase (EC 1.1.1.27), malate dehydrogenase (EC 1.1.1.37), aspartate aminotransferase (EC 2.6.1.1), alanine aminotransferase (EC 2.6.1.2), and $\gamma$-glutamyl transpeptidase (EC 2.3.2.1) was studied in the mouse and in the guinea pig. In both species enzymes are released from thrombocytes into the serum during clotting. The extent of the enzyme liberation is such that activity measurements in serum are of restricted or no diagnostic value, if high activities of the respective enzymes are also present in the thrombocytes (e. g. lactate and malate dehydrogenase). It appears that enzymes with lower molecular weights are released at a slightly faster rate than those with higher molecular weights. $\gamma$-Glutamyl transpeptidase is set free from guinea pig thombocytes despite its firm binding to cell structures. The enzyme is either solubilized during the clotting process, or subcellular particles are liberated to which the enzyme is attached.

Wie wir kürzlich berichtet haben, treten bei der Blutgerinnung Stoffwechselenzyme aus Thrombozyten in das Blutserum über (1). Unsere Untersuchungen betrafen den Menschen, den Hund, das Kaninchen und die Ratte und zeigten außerordentliche Speziesunterschiede im Ausmaß des Enzymaustritts. Bei der Gerinnung von Menschenblut ist der Austritt von Enzymen aus Thrombocyten gering, und in der klinischen Routine können die Aktivitäten im Serum und im Plasma ohne größeten Fehler einander gleichgesetzt werden. Bei den Versuchstieren hingegen und besonders ausgeprägt bei der Ratte, werden derart große Mengen von Enzymen aus Thrombocyten freigesetzt, $\mathrm{da} B$ die Aktivitätsbestimmungen im Serum in ihrer diagnostischen Aussagekraft wertlos sind, es sei denn

1. es handelt sich um Enzyme, die nicht oder in sehr geringen Mengen in Thrombocyten vorkommen;

2. das Serum wird aus Nativ-Plasma gewonnen (siehe Methoden).

In der Zwischenzeit haben wir zwei weitere wichtige Versuchstiere untersucht: die Maus und das Meerschweinchen. Wie schon in der vorausgegangenen Ar-

1) Mit Unterstützung des U. S. Public Health Service, Grant HE 03912 und Career Development Award 5-K3-GM-15524 (H. M.). 2) Research Fellow der Deutschen Forschungsgemeinschaft. beit studierten wir den Einfluß der Blutgerinnung auf die Aktivitäten der Lactatdehydrogenase, der Malatdehydrogenase, der Aspartataminotransferase und der Alaninaminotransferase. Zusätzlich untersuchten wir die $\gamma$-Glutamyltranspeptidase, ein Enzym, das in neuerer Zeit zunehmende Bedeutung in der Diagnostik von Lebererkrankungen erlangt.

\section{Methodik}

Das Untersuchungsmaterial wurde in Plastikgefäßen mit unbenetzbarer innerer Oberfläche gewonnen, aufgearbeitet und aufbewahrt. Falls nicht anders vermerkt, wurden die Versuche bei Raumtemperatur durchgeführt.

\section{Gerinnungsversuche}

Gesunden Meerschweinchen und Mäusen wurde nach leichtem Atherrausch mittels Herzpunktion Blut cntnommen und sofort auf jeweils vier Plastikröhrchen verteilt. Eins dieser Röhrchen wurde unmittelbar nach der Blutentnahme, die restlichen nach 30 Min., 60 Min. bzw. 120 Min. für 2 Min. mit $15000 \mathrm{~g}$ zentrifugiert. Das übcrstehende zellfreie Nativ-Plasma in dem zuerst zentrifugierten Röhrchen wurde sofort separiert und im Anschluß an dic Nachgerinnung erneut zentrifugiert; das dann überstehende Serum wurde separiert. Die weiteren Blutproben waren ausnahmslos vor dem Zentrifugicren geronnen, und Serum konnte wie üblich gewonnen werden.

Erythro- und Thrombocyten

Die Gewinnung und Aufarbeitung der Blutzellen haben wit ausführlich beschrieben (1). Die Zellen wurden mit Digitonin aufge- 
schlossen und die Enzymaktivitäten im klaren Uberstand (Zentrifugieren für $10 \mathrm{Min}$. mit $16000 \mathrm{~g}$ bei $4^{\circ}$ ) bestimmt. Die $\gamma$-Glutamyltranspeptidase-Aktivitäten wurden, sofern nicht anders vermerkt, im Gesamt-Lysat gemessen.

\section{Meßmethoden}

Folgende Enzyme wurden untersucht:

Lactatdehydrogenase (EC 1.1.1.27)

Malatdehydrogenase (EC 1.1.1.37)

Aspartataminotransferase $=$ Glutamat-oxalacetat-transaminase

(EC 2.6.1.1)

Alaninaminotransferase $=$ Glutamat-pyruvat-transaminase

(EC 2.6.1.2)

$\gamma$-Glutamyltranspeptidase (EC 2.3.2.1)

Dehydrogenasen und Transaminasen wurden im optischen UV-

Test und $\gamma$-Glutamyltranspeptidase kolorimetrisch (Wellenlänge

$405 \mathrm{~nm}$ ) bestimmt. Die Aktivitätsmessungen wurden bei $25^{\circ} \mathrm{mit}$ Mikroliter-Methoden (2) im Photometer Eppendorf mit automatischem Küvettenwechsler und Registriereinrichtung durchgeführt. Die Enzymaktivität ist angegeben für Serum in $\mathrm{mU} / \mathrm{ml}$, für Erythrocyten in U/g Hämoglobin ( $\mathrm{Hb})$ und für Thrombocyten in $U / 10^{11}$ Zellen. Eine U entspricht der Enzymmenge, die $1 \mu \mathrm{Mol}$ Substrat pro Minute umsetzt.

Testansätze (Endkonzentrationen):

Lactatdehydrogenase: Triäthanolamin-Puffer, $\mathrm{pH}$ 7,5, $50 \mathrm{~mm}$; NADH 0,35 mm; Pyruvat 0,55 mM.

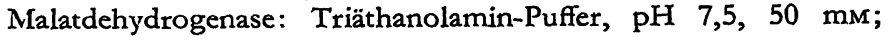
NADH $0,35 \mathrm{mar}$; Oxalacetat 2,0 mis.

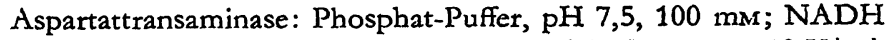
$0,50 \mathrm{~mm} ; 2-$ Oxoglutarat $10.5 \mathrm{~mm}$; Malatdehydrogenase $10 \mathrm{U} / \mathrm{ml}$; i-Asparartat $50 \mathrm{~mm}$.

Alanintransaminase: Phosphat-Puffer, $\mathrm{pH} 7,5,100 \mathrm{~mm}$; NADH $0,50 \mathrm{~mm} ; 2$-Oxoglutarat $10 \mathrm{~mm}$; Lactatdehydrogenase $5 \mathrm{U} / \mathrm{ml}$; L-Alanin $80 \mathrm{~mm}$.

$\gamma$-Glutamyltranspeptidase: Tris-Puffer, $\mathrm{pH} 8,5,100 \mathrm{~mm} ; \boldsymbol{\gamma}$-Glutamyl-p-nitroanilid 3,5 mm; Glycyl-glycin $50 \mathrm{~mm}$.

Die Endvolumina betrugen um $300 \mu l$, je nach Enzymaktivität wurden $1-20 \mu l$ von der zu untersuchenden Probe eingesetzt.

\section{Ergebnisse und Diskussion}

Die Ergebnisse der Gerinnungsversuche mit Blut der Maus sind in Tabelle 1 zusammengestellt. Die von uns gemessenen Ausgangsaktivitäten liegen in der gleichen Größenordnung wie die von ZIMMERManN und Mit-

Tab. 1

Enzymaktivitäten im Serum der Maus in Abhängigkeit von der Zeitspanne zwischen Blutentnahme und Serumgewinnung

\begin{tabular}{cccccc}
\hline $\begin{array}{c}\text { Zeit } \\
\text { (Min.) }\end{array}$ & $\begin{array}{c}\text { Lactat- } \\
\text { dehydro- } \\
\text { genase }\end{array}$ & $\begin{array}{c}\text { Malat- } \\
\text { dehydro- } \\
\text { genase }\end{array}$ & $\begin{array}{c}\text { Aspartat- } \\
\text { trans- } \\
\text { aminase }\end{array}$ & $\begin{array}{c}\text { Alanin- } \\
\text { trans- } \\
\text { aminase }\end{array}$ & $\begin{array}{c}\gamma \text {-Glutamyl- } \\
\text { trans- } \\
\text { peptidase }\end{array}$ \\
\hline \multicolumn{7}{c}{$\left(\mathrm{mU} / \mathrm{ml}, 25^{\circ}, \overline{\mathrm{x}} \pm \mathrm{s}\right)$} \\
$0-1$ & $316 \pm 98$ & $284 \pm 99$ & $44,7 \pm 14,8$ & $16,8 \pm 2,0$ & 0,0 \\
30 & $603 \pm 161$ & $410 \pm 113$ & $44,5 \pm 13,3$ & $16,6 \pm 2,8$ & 0,0 \\
60 & $764 \pm 355$ & $499 \pm 199$ & $45,6 \pm 13,1$ & $16,8 \pm 2,9$ & 0,0 \\
120 & $1030 \pm 426$ & $669 \pm 224$ & $51,5 \pm 20,2$ & $17,0 \pm \pm 2,5$ & 0,0 \\
& $\mathrm{n}=10$ & $\mathrm{n}=10$ & $\mathrm{n}=9$ & $\mathrm{n}=5$ & $\mathrm{n}=5$ \\
\hline
\end{tabular}

arbeitern (3) angegebenen Enzymaktivitäten im Plasma der Maus. Geringfügige Unterschiede sind durch verschiedenartige Methodik bedingt.

Im Verlaufe der Blutgerinnung nahmen die Aktivitäten der Lactat- und Malatdehydrogenase im Serum erheblich zu. Die Aktivität der Aspartattransaminase blieb während der ersten Stunde praktisch unverändert, um nach zwei Stunden gering anzusteigen. Die Aktivität der Alanintransaminase blieb während des gesamten
Beobachtungszeitraumes praktisch unverändert. $\gamma$-Glutamyltranspeptidase-Aktivität war in keiner der untersuchten Serumproben nachweisbar.

Die beobachteten Aktivitätsanstieg'e sind denjenigen sệ ähnlich, die wir bereits für die Ratte beschrieben haben. Auch konnten wir durch Gerinnungsversuche mit thrombocytenreichem Rattenplasma und einem Vergleich der Enzymmuster in den Blutzellen mit dem Muster des Aktivitätsantieges im Serum zeigen, daß die Enzyme bei der Blutgerinnung aus Thrombocyten austreten (1).

Enzymaustritt aus Thrombocyten während der Gerinnung dürfte auch die nun beobachteten Anstiege von Enzymaktivitäten im Serum der Maus erklären. Um diese Hypothese zu prüfen, haben wir die Aktivitäten der von uns untersuchten Enzyme in Erythrocyten und Thrombocyten bestimmt. Die Ergebnisse sind in Tabelle 2 zusammengestellt. Die Enzymmuster in den bei-

Tab. 2

Enzymaktivitäten in Erythrocyten und Thrombocyten der Maus

\begin{tabular}{|c|c|c|c|c|c|}
\hline & $\begin{array}{l}\text { Lactat- } \\
\text { dehydro- } \\
\text { genase }\end{array}$ & $\begin{array}{l}\text { Malat- } \\
\text { dehydro- } \\
\text { genase }\end{array}$ & $\begin{array}{l}\text { Aspartat- } \\
\text { trans- } \\
\text { aminase }\end{array}$ & $\begin{array}{l}\text { Alanin- } \\
\text { trans- } \\
\text { aminase }\end{array}$ & $\begin{array}{c}\gamma-\text { Glutamyl- } \\
\text { trans- } \\
\text { peptidase }\end{array}$ \\
\hline \multicolumn{6}{|c|}{ (U/g Hb, $\left.25^{\circ}, \dot{x} \pm s, n=5\right)$} \\
\hline $\begin{array}{c}\text { Erythro- } \\
\text { cyten }\end{array}$ & $208 \pm 27,5$ & $108 \pm 7,8$ & $1,3 \pm 1,1$ & $0,36 \pm 1,0$ & $0,69 \pm 0,44$ \\
\hline \multicolumn{6}{|c|}{$\left(U / 10^{11}\right.$ Zellen, $\left.25^{\circ}, \bar{x} \pm s, n=5\right)$} \\
\hline $\begin{array}{l}\text { Thrombo- } \\
\text { cyten }\end{array}$ & $329 \pm 34,3$ & $115 \pm 28,9$ & $2,8 \pm 0,8$ & $0,82 \pm 0,4$ & $0,1 \pm 0,1$ \\
\hline
\end{tabular}

den Blutzelltypen sind sich ähnlich und unterscheiden sich auch kaum vom Muster des Aktivitätsantieges im Serum. Der Quotient Lactat-/Malatdehydrogenase beträgt z. B. in Erythrocyten 1,9, in Thrombocyten 2,85. Der gleiche Quotient für die Aktivitätszunahme im Serum beträgt nach 30 Min. 2,5, nach 60 Min. 2,1 und nach 120 Min. 1,85. Wenn die im Serum nach der Blutgerinnung erhöht gefundenen Enzymaktivitäten ausschließlich aus Thrombocyten stammen, wie wir vermuten, dann müßte die Malatdehydrogenase proportional stärker aus Thrombocyten austreten als die Lactatdehydrogenase. Das weit geringere Molekulargewicht der Malatdehydrogenase (67000 im Vergleich zu etwa 120000 für Lactatdehydrogenase) könnte uns hierfür eine Erklärungsmöglichkeit bieten, wissen wir doch, daß Enzyme aus der experimentell geschädigten Leberżelle entsprechend ihren Molekulargewichten austreten (4).

Einen geringen Beitrag von Erythrocyten-Enzymen können wir nicht mit Sicherheit ausschließen, da wir das Hämoglobin im Serum als natürlichen „Marker“" für Erythrocyten-Zerstörung nicht gemessen haben. Hämolytische Seren wurden aber verworfen. Der Einfluß einer unsichtbaren Hämolyse auf beispielsweise die Lactatdehydrogenase-Aktivitäten kann nicht sehr groß gewesen sein, wenn man bedenkt, da $1 \mathrm{mg} \mathrm{Hb}$ in Erythrocyten der Maus etwa $200 \mathrm{mU}$ Lactatdehydrogenase entspricht (Tab. 2). Ein Hämolysegrad von 0,3 mg $\mathrm{Hb} / \mathrm{m} l$ Serum kann mit bloßem Auge mit Sicherheit erkannt werden. Eine derartige Hämolyse würde 
einhergehen mit einer Freisetzung von $0,3 \cdot 200=60$ $\mathrm{mU}$ Lactatdehydrogenase $/ \mathrm{m} l$ Serum. In den ersten 30 Min. der Gerinnung beobachteten wir jedoch schon eine fast 5 fach stärkere Zunahme der Lactatdehydrogenase-Aktivität ohne sichtbare Hämolyse.

In den Tabellen 3 und 4 sind die Ergebnisse unserer Versuche mit Meerschweinchen zusammengefaßt. Wir

Tab. 3

Enzymaktivitäten im Serum des Meerschweinchens in Abhängigkeit von der Zeitspanne zwischen Blutentnatime und Serumgewinnung

\begin{tabular}{|c|c|c|c|c|c|}
\hline $\begin{array}{c}\text { Zeit } \\
\text { (Min.) }\end{array}$ & $\begin{array}{l}\text { Lactat- } \\
\text { dehydro- } \\
\text { genase }\end{array}$ & $\begin{array}{l}\text { Malat- } \\
\text { dehydro- } \\
\text { genase }\end{array}$ & $\begin{array}{l}\text { Aspartat- } \\
\text { trans- } \\
\text { aminase }\end{array}$ & $\begin{array}{l}\text { Alanin- } \\
\text { trans- } \\
\text { aminase }\end{array}$ & $\begin{array}{c}\gamma \text {-Glutamyl } \\
\text { trans- } \\
\text { peptidase }\end{array}$ \\
\hline \multicolumn{6}{|c|}{$\left(\mathrm{mU} / \mathrm{ml}, 25^{\circ}, \overline{\mathrm{x}} \pm \mathrm{s}\right)$} \\
\hline $\begin{array}{c}0-1 \\
30 \\
60 \\
120\end{array}$ & $\begin{array}{r}63,3 \pm 18,9 \\
99,0 \pm 30,8 \\
109 \pm 24,7 \\
136 \pm 26,7 \\
n=9\end{array}$ & $\begin{array}{c}205 \pm 34,4 \\
235 \pm 45,7 \\
249 \pm 37,9 \\
281 \pm 37,3 \\
n=9\end{array}$ & $\begin{array}{c}21,6 \pm 5,9 \\
21,5 \pm 5,2 \\
21,6 \pm 5,3 \\
22,1 \pm 5,8 \\
n=8\end{array}$ & $\begin{array}{c}21,7 \pm 3,4 \\
21,8 \pm 3,8 \\
21,7 \pm 4,0 \\
22,1 \pm 3,6 \\
n=8\end{array}$ & $\begin{array}{c}2,8 \pm 0,7 \\
4,6 \pm 1,7 \\
6,9 \pm 2,2 \\
8,7 \pm 2,1 \\
n=8\end{array}$ \\
\hline
\end{tabular}

Tab. 4

Enzymaktivitäten in Erythrocyten und Thrombocyten des Meerschweinchens

\begin{tabular}{|c|c|c|c|c|c|}
\hline & $\begin{array}{l}\text { Lactat- } \\
\text { dehydro- } \\
\text { genase }\end{array}$ & $\begin{array}{c}\text { Malat- } \\
\text { dehydro- } \\
\text { genase }\end{array}$ & $\begin{array}{l}\text { Aspartat- } \\
\text { trans- } \\
\text { aminase }\end{array}$ & $\begin{array}{c}\text { Alanin- } \\
\text { trans- } \\
\text { aminase }\end{array}$ & $\begin{array}{c}\gamma \text {-Glutamyl- } \\
\text { trans- } \\
\text { peptidase }\end{array}$ \\
\hline \multicolumn{6}{|c|}{$\left(\mathrm{U} / \mathrm{g} \mathrm{Hb}, 25^{\circ}, \overline{\mathrm{x}} \pm \mathrm{s}, \mathrm{n}=6\right)$} \\
\hline cyten & $125 \pm 14,0$ & $139 \pm 22,0$ & $0,78 \pm 0,45$ & $0,38 \pm 0,41$ & $0,34 \pm 0,12$ \\
\hline \multicolumn{6}{|c|}{$\left(\mathrm{U} / 10^{11}\right.$ Zellen, $\left.25^{\circ}, \overline{\mathrm{x}} \pm \mathrm{s}, \mathrm{n}=8\right)$} \\
\hline cyten & $161 \pm 30,0$ & $106 \pm 28,5$ & $3,5 \pm 0,80$ & $1,6 \pm 0,66$ & $17,8 \pm 3,8$ \\
\hline
\end{tabular}

finden hier im Verlaufe der Gerinnung im Vergleich zur Maus einen deutlich geringeren Anstieg der Lactatdehydrogenase- und Malatdehydrogenase-Aktivitäten im Serum. Die Aktivitäten der Transaminasen verändern sich während des Beobachtungszeitraumes nur unbedeutend. Die $\gamma$-Glutamyltranspeptidase-Aktivität nimmt von einem Ausgangswert von $2,8 \mathrm{mU} / \mathrm{ml}$ innerhalb von 2 Stdn. auf $8,7 \mathrm{mU} / \mathrm{m} l$ zu. Der Vergleich der beobachteten Aktivitätsanstiege mit den Enzymmustern in Erythrocyten und Thrombocyten zeigt auch hier, daß Thrombocyten mit großer Wahrscheinlichkeit als Quelle für die ausgetretenen Enzyme anzusehen sind. Wir finden nämlich beim Meerschweinchen $\gamma$-Glutamyltranspeptidase mit relativ hoher Aktivität in Thrombocyten, in Erythrocyten ist sie hingegen kaum nachweisbar. Der Quotient Lactat-/Malatdehydrogenase zeigt uns wieder, $\mathrm{da} B$ die Malatdehydrogenase proportional stärker austritt als die Lactatdehydrogenase mit ihrem höheren Molekulargewicht. Da wir auch in diesen Versuchen die Hämolyse nicht gemessen haben, können wird einen geringen Beitrag von Erythrocyten nicht mit Sicherheit ausschließen.

Nachdem der Austritt von Stoffwechselenzymen aus Thrombocyten bei der Blutgerinnung nun auch bei der Maus und beim Meerschweinchen bestätigt ist, ist es dringend zu empfehlen, bei Versuchen mit anderen Tierspezies den Einfluß der Gerinnung auf die Enzymaktivitäten im Serum zu prüfen. So fanden wir kürzlich z. B. auch bei der Katze sehr erhebliche Akti- vitätsanstiege der Lactatdehydrogenase und Malatdehydrogenase während der Gerinnung. Sofern die quantitativen Enzymmuster in den Thrombocyten einer Tierspezies bekannt sind, kann man die zu erwartenden gerinnungsbedingten Aktivitätsanstiege im Serum abschätzen, nachdem das Ausmaß des Enzymaustritts für ein Enzym, am besten Lactatdehydrogenase, bestimmt worden ist. Eine Veröffentlichung hierüber haben wir in Vorbereitung.

Der Einfluß der Gerinnung kann leicht umgangen werden, indem das Serum aus Nativ-Plasma gewonnen wird, wie wir es im methodischen Teil beschrieben haben. Messungen im Plasma sind dann zulässig, wenn ein Einfluß des Antikoagulans auf das zu messende Enzym und auf Hilfs- und Indikatorenzyme in gekoppelten Tests und auf die Blutzellen (z. B. osmotische Wasserverschiebungen aus Blutzellen und Plasma) ausgeschlossen werden können. Wir fanden jedoch auch im Heparin-Plasma der Ratte, im Vergleich zur Gerinnung allerdings sehr leichte Aktivitätsanstiege für Lactatdehydrogenase, Malatdehydrogenase und Aspartattransaminase (1).

Es ist gegenwärtig nicht möglich, eine Aussage zu machen über den Mechanismus des Austritts von Stoffwechselenzymen aus Thrombocyten. Während der verschiedenen Blutgerinnungsphasen kommt es zu weitgehenden morphologischen Veränderungen der Blutplättchen. Von einigen Autoren wird hierbei eine teilweise Auflösung, von anderen Erhaltung der äußeren Plättchenmembran beschrieben. Hierüber hat Hovig (5) in einer ausführlichen Übersicht referiert. DAVEY und LüsCHER (6) fanden bei der Inkubation von menschlichen Thrombocyten mit Thrombin, daß niedermolekulare Substanzen (Nucleotide, Serotonin und Aminosäuren) ebenso wie verschiedene Proteine, darunter saure Hydrolasen, selektiv aus den Plättchen austreten; zu einer allgemeinen Steigerung der Membranpermeabilität kommt es nicht. Von den sauren Hydrolasen wird relativ mehr $\beta$-Glucuronidase (EC 3.2.1.31) als saure Phosphatase (EC 3.1.3.2.) freigesetzt. Dabei scheinen Unterschiede in der Lokalisation der beiden Enzyme in subzellulären Strukturen ( $\alpha$-Granula und Lysosome) eine Rolle zu spielen. Ähnliche Resultate wurden von Holmsen und DAY (7) mitgeteilt. Die außerdem gemessenen Enzyme Lactathydrogenase und Cytochrom-c Oxydase (EC 1.9.3.1) wurden nicht in nennenswerten Mengen freigesetzt. Unsere Beobachtungen über den geringen Grad des Austritts von Lactatdehydrogenase, Malatdehydrogenase und Aspartattransaminase aus menschlichen Thrombocyten (1) stimmen recht gut mit diesen Ergebnissen überein. Der starke Enzymaustritt bei den Versuchstieren und die ausgeprägten Speziesunterschiede lassen sich jedoch nicht erklären.

Die Freisetzung von $\boldsymbol{\gamma}$-Glutamyltranspeptidase aus Thrombocyten des Meerschweinchens bei der Blutgerinnung hat uns überrascht, da dieses Enzym in Geweben stark strukturgebunden ist $(8,9)$ und beim Homogenisieren nur zu cinem geringen Teil extrahiert werden kann. Die Aktivität wird deshalb im allgemeinen im 
Gesamthomogenat gemessen. Auch nach unserer Erfahrung mit verschiedenen Spezies gehen selbst bei intensivstem Homogenisieren (Ultraturrax) von NierenCortex, dem Gewebe mit der höchsten $\gamma$-Glutamyltranspeptidase-Aktivität, nur $2-10 \%$ des aktiven Enzymproteins in Lösung.

Um die Möglichkeit ausschließen zu können, daß die $\gamma$-Glutamyltranspeptidase in Thrombocyten des Meerschweinchens in einer löslichen Form vorliegt, haben wir in fünf Versuchen die Aktivität sowohl im GesamtLysat als auch im Extrakt (aufgearbeitet wie zur Bestimmung der restlichen Enzymaktivitäten) gemessen. Wir fanden dabei im Extrakt $0,98 \pm 0,62$ und im Gesamt-Lysat 17,7 $\pm 4,3 \mathrm{U} / 10^{11}$ Zellen (Mittelwerte \pm Standardabweichung). Wir schließen daraus, daß die $\gamma$-Glutamyltranspeptidase in Thrombocyten ebenfalls in fester Strukturbindung vorliegt.

Wenn die $y$-Glutamyltranspeptidase trotz ihrer starken Strukturbindung zusammen mit anderen leicht extrahierbaren Enzymen bei der Blutgerinnung aus Throm- bocyten ins Blutserum übertritt, so muß entweder das Enzymprotein im Verlauf des Gerinnungsvorganges aus seiner Strukturbindung gelöst werden oder aber es werden subzelluläre Partikel, an die das Enzym gebunden ist, aus den Zellen freigesetzt. Die Partikel wären entsprechend klein, so daß sie mit der von uns gewählten Methodik beim Zentrifugieren nicht aus dem Serum entfernt werden.

Es sei erwāhnt, daß die $\gamma$-Glutamyltranspeptidase mit nennenswerter Aktivität nur in den Thrombocyten des Meerschweinchens vorzukommen scheint. In anderen von uns untersuchten Spezies - Mensch, MarmosetAffe, Hund, Kaninchen, Ratte und Maus - ist das Enzym in den Thrombocyten nicht oder nur mit sehr geringer Aktivität nachweisbar (R. FRIEDEL, $H$. MATTENHEIMER, G. FORSTER, unveröffentlicht).

Die Autoren danken Mrs. Henny DeBruin, Miss Ludmilla Demidow, M. S., Mrs. Elke Goegcke und Mrs. Zofia Pasnick für unermüdliche und exakte Mitarbeit.

\section{Literatur}

1. Friedel, R. und H. Mattenheimer, Clin. chim. Acta (Amsterdam) 30, 37 (1970). - 2. Mattenhermer, H., Mikromethoden für das klinisch-chemische und biochemische Laboratorium, 2. Auflage, Walter de Gruyter, Berlin (1966). - 3. ZnMmermanN, H. J., M. A. Schwartz, L. E. Boley und M. West, J. Labotat. Clin. Med., S. Louis 66, 961 (1959). - 4. Schmmd, E., F. W. SChmid, C. Herfarth, K. OptTz und W. Vogell, Enzymol. biol. clin. 7, 185 (1966). - 5. Hovrg, T., Series Haematologica 1/2, 3 (1968). - 6. Davey, M. G. und E. F. Lüscher, Biochim. biophysica Acta (Amsterdam) 165, 490 (1968). - 7. Hólmsen, H. und H. J. DAY, Nature (London) 129, 760 (1968). - 8. SzEWCZUK, A. und T. Baranowskr, Biochem. Z. 338, 317 (1963). - 9. Szewczuk, A., Clin. chim. Acta (Amsterdam) 14, 608 (1966).

\author{
Dr. Rainer Friedel \\ Abteilung für Klinische Biochemie \\ Medizinische Hochschule Hannover \\ 3 Hannover, Roderbruchstr. 101 \\ Prof. Dr. H. Mattenheimer \\ Rush-Presbyterian-St. Luke's Medical Center \\ 1753 W. Congress Parkway \\ Chicago 60612, Illinois, USA
}

